\title{
XIPHINEMA CAMPINENSE, NOVA ESPÉCIE (NEMATODA, DORYLAIMIDAE)
}

\author{
Luís Gonzaga E. Lordeino \\ Engenheiro agronomo, Assistente da Cadeira de Zoologia da Escota Superior de Agri- \\ cultura "Luis de Queiroz", Universidade de São Paulo
}

Durante os nossos exames de material de planta colhido na Estação Experimental Central do Instituto Agronômico, temos obtido grupos de Nematoda do gênero Xiphinema, constituídos quase que exclusivamente de larvas. $O$ material (solo e raízes) é invariàvelmente tratado segundo o Método do Funil de Baermann, os espécimes fixados pela formalina a $6 \%$ e os desenhos feitos com o auxílio da câmara clara.

Tendo estudado fêmeas adultas coligidas em fevereiro de 1951 e tendo-as como filiadas a uma espécie nova para a ciência, deliberamos descrevê-la. Com isso, elevam-se a duas, as espécies de Xiphinema referidas para a fauna do Brasil : $X$. brasiliense Lordello, 1951 (forma opistodélfica, parasita de raízes e tubérculos de batatinha, (Solcnum tuberosum L.) (5), e $X$. campinense (espécie anfidélfica), que descrevemos a seguir.

Xiphinema campinense, $\mathbf{n}$. sp.

$q=2.517$ micros $; \quad \mathbf{a}=60,9 ; \quad \mathbf{b}=6,2 ; \mathbf{c}=33,1-34,5 ; \quad \mathbf{V}=39,8-40,3 \%$

Cabeça separada do corpo por apenas uma leve depressão da cutícula ; lábios fundidos, com dois círculos de papilas diminutas. Cutícula completamente lisa, sem nenhuma estriação transversal. Anfídio curto e largo.

Comprimento do estilete, no adulto, 154-161 micros, dos quais 90-97 micros representam a ponta que é substituída por ocasião da ecdise.

A porção anterior do esôfago é dada por um tubo flexível e fino que, ao se expandir na base, produz o bulbo terminal alongado, em cujo extremo anterior se localiza o núcleo da glândula esofagiana dorsal.

$\mathrm{O}$ poro excretor não foi localizado. $\mathrm{O}$ anel nervoso perisofágico situa-se logo abaixo da base do estilete, quando êste se apresenta inteiramente recolhido.

Cárdia obscuro. A vulva é representada por uma fenda transversal localizada a 923-935 micros da frente da cabeça; a vagina se estende pelo corpo até quase a metade de sua largura. Dois ovários, um anterior e outro posterior em relação à vulva, ambos bastante curtos. Apenas dois ovos são vistos, prestes a serem lançados para o exterior, um correspondente a cada ovário. Medem 154-149 micros de comprimento por 26,4-28,0 micros de largura, representando o seu diâmetro até $73 \%$ da largura do corpo, tomada na altura da vulva. 
Tal como referimos ao descrever $X$. brasiliense (5), as avantajadas dimensões do ôvo e o seu pequeno número nos ovários são bons indícios de um ciclo vital longo.

O corpo atenua-se suavemente para as extremidades, principalmente para a posterior, dando origem a uma cauda afilada, com 67-70 micros de comprimento.

As papilas caudais são em número de quatro em cada face lateral, dispostas segundo a ilustração que apresentamos.

Os machos são desconhecidos; apenas poucas fêmeas e muitas larvas foram encontradas.

As larvas - A forma da cauda é semelhante à dos adultos. As mensurações obtidas do exemplar cuja extremidade caudal fazemos figurar no presente trabalho foram, em micros, as seguintes : comprimento - 1.525 ; largura ao nível do meio do corpo - 39,6 ; estilete - 127,6 ; extremidade trocável -88 ; comprimento do esôfago - 303,6; bulbo terminal - 92,4.

Inimigo natural - Constatamos uma larva cujo corpo se apresentava completamente tomado por Sporozoa da Ordem Microsporidia. O diâmetro do parasita andou por volta de 5 micros.

Pátria - Fazenda Santa Elisa (Estação Experimental Central do Instituto Agronômico), Campinas, Estado de São Paulo, ao redor de raízes de algumas plantas de interêsse econômico, para as quais representa um ecto-parasita, a saber: guandu (Cajanus indicus Spreng.), soja (Glycine $\max$ (L.) Merr.), repôlho (Brassica oleracea L., var. capitata L.) e arroz (Oryza sativa L.).

As raízes de arroz que examinamos foram colhidas em fevereiro de 1951, de um experimento de adubação da Secção de Cereais e Leguminosas do Instituto Agronômico, em seu 6. ${ }^{\circ}$ ano de cultura. Para o desenvolvimento péssimo das plantas, sem dúvida, contribuía a abundância de larvas coligidas ao redor de suas raízes.

\section{DIAGNOSE}

Comparamos $X$. campinense com as demais espécies anfidélficas conhecidas $(2,3,4,6,7)$. A espécie mais próxima é $X$. pratense Loos, 1949 (1), descrita no Ceilão. As diferenças observadas (dimensões, ausência de qualquer estriação cuticular, número e disposição das papilas caudais) justificam o estabelecimento da nova espécie.

\section{DIAGNOSIS}

A X. pratensi Loos, 1949, cui affinis est, differt cuticula haud striata, numero dispusitioneque papillarum caudae necnon dimensionibus.

\section{SUMMARY}

$X$. campinense n. sp. is described. It is a Xiphinema closely resembling $X$. pratense Loos, 1949, from which it is distinguished by its measurements, by the absence of any cuticular transverse striation as well as by the number and location of the caudal papillae.

(1) A ortografia original da espécie descrita por Loos (4) 6 Xiphinema pratensis, que emendamos para $X$ iphinema pratense, por refletir evidentemente um érro tipográfico ou um lapsus calami (art. 19.8 das Regras Intermacionais de Nomenclatura Zoologiea, 1). 


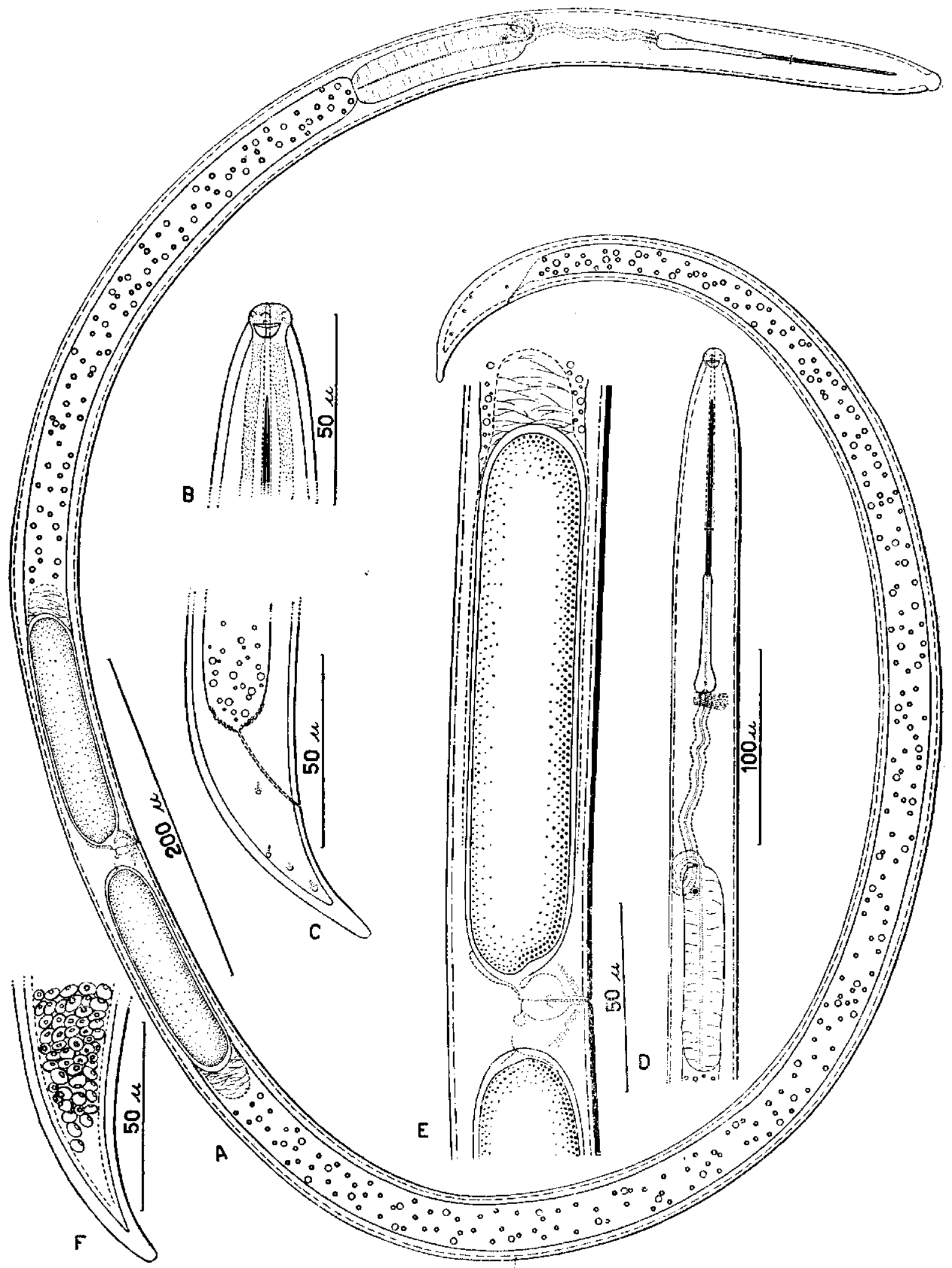

FIGLra 1.-Xiphinema campinense n. sp.; $A$ - fêmea adulta; $B$ - detalhe da cabeça; $C$ - extremidade caudal do adulto; $D$ - detalhe da região do esôfago; $E$ - idem, da região dos ovários; $F$ - extremidade caudal de uma larva parasitada por Sporozoa da Ordem Microsporidia. 


\section{LITERATURA CITADA}

1. Amaral, Afránio do. Codificaçāo da nomenclatura zoológica. Arq. de Zoo. do Est. de S. Paulo 7: 379-437. 1950.

2. Cobb, N. A. New nematode genera found inhabiting freshwater and nonbrackish soils. Jour. Wash. Acad. of Sci. 3: 132-144, fig. 1, est. 1. 1913.

3. Goodey, T. $E m$ Soil and freshwater nematodes, a monograph, pág. I-XXVI + 1-390, fig. 1-90, Methuen \& Co. 1951.

4. Loos, C. A. Notes on free-living and plant parasitic nematodes of Ceylon - N.॰ 5 . Jour. Zool. Soc. of India 1: 23-29, fig. 1-5. 1949.

5. Lordello, Luís Gonzaga E. Xiphinema brasiliense, nova espécie de nematóide do Brasil, parasita de Solanum tuberosum L. Bragantia 11: 87-90, fig. 1. 1951.

6. Thorne, G. A monograph of the nematodes of the superfamily Dorylaimoidea. Capita Zoo. 8: 1-261, est. 1-32. 1939.

7. Thorne, Gerald e Merlin W. Allen. Paratylenchus hamatus n. sp. and Xiphinema index n. sp., two nematodes associated with fig roots, with a note on Paratylenchus anceps Cobb. Proc. of the Helm. Soc. of Wash. 17: 27-35, fig. 1-4. 1950. 